\title{
ARTICLE OPEN \\ A flexible solar-blind 2D boron nitride nanopaper-based photodetector with high thermal resistance
}

\author{
Chun-Ho Lin ${ }^{1}$, Hui-Chun Fu' ${ }^{1}$ Bin Cheng ${ }^{1}$, Meng-Lin Tsai ${ }^{1}{ }^{1}$, Wei Luo ${ }^{2}$, Lihui Zhou ${ }^{2}$, Soo-Hwan Jang ${ }^{2}$, Liangbing Hu ${ }^{2}$ and Jr-Hau He ${ }^{1}$
}

Flexible electronics are expected to play a key role in connecting human lives with versatile smart electronic devices due to their adaptability to different shapes, surfaces, and even the human body. However, heat management issues found in most flexible devices due to the low thermal conductivity of conventional plastic or paper substrates become significant for large-scale integration or high-temperature applications. In this study, we employed high thermal conductivity nanopaper composed of twodimensional (2D) hexagonal boron nitride nanosheets and one-dimensional nanofibrillated cellulose to form a flexible deepultraviolet photodetector demonstrating superior photodetectivity of up to $8.05 \times 10^{10} \mathrm{~cm} \mathrm{~Hz} z^{1 / 2} / \mathrm{W}$, a short response time of $0.267 \mathrm{~s}$, and excellent flexible durability featuring repeatable ON/OFF photoswitching over 200 bending cycles. Because the boron nitride paper has a high thermal conductivity of $146 \mathrm{~W} / \mathrm{mK}$, which is three orders of magnitude larger than plastic or paper substrates, the photodetectors can work at high temperatures of up to $200^{\circ} \mathrm{C}$. The boron nitride paper-based strategy described herein suggests a path for improving heat dissipation in flexible electronics and achieving high-performance deep-ultraviolet photodetectors, which can be applied in wearable applications.

npj 2D Materials and Applications (2018)2:23; doi:10.1038/s41699-018-0070-6

\section{INTRODUCTION}

Flexible electronics are developing rapidly, incorporating all kinds of functional attributes into pliable substrates to meet the personalized demands of future technology, such as wearable electronics, the internet of things, electronic skins, and biomedical healthcare, which are expected to bring unprecedented innovations to our lives. ${ }^{1-5}$ Due to the unique capabilities of these devices to stretch, bend, twist, and fold, thus conforming to all kinds of applications, flexible electronics hold a market size of around $\$ 6$ billion as of 2017 , which is expected to grow to $\$ 33$ billion by $2024 .^{6}$

Flexible electronics can be classified into two categories: plasticand paper-based devices. ${ }^{7}$ In the past few years, plastic-based devices have been the mainstream for commercial flexible applications, which are fabricated by mounting electronic components on flexible polymer substrates, such as polyimide, polyethylene terephthalate, and polydimethylsiloxane. Due to the soft nature and flat surface of plastic substrates, complex circuit assemblies can be manufactured by the same printing technology used to produce rigid printed circuit boards, thus achieving mass production for plastic electronics. ${ }^{2}$ With single-sided circuit design and the capability to easily integrate multiple printed assemblies, plastic-based devices are ideal for dynamic and high-flex applications. Nevertheless, there are still several drawbacks for plastic devices, including repair difficulties, environmental unfriendliness, and high production costs.

On the other hand, paper devices (or fiber-based, in a broad sense) are lightweight, foldable, low-cost, and easy to process compared to plastic-based materials. ${ }^{8}$ Furthermore, because of their biodegradable and recyclable nature, paper devices meet the trends of next-generation green technology, resulting in rapid market-size growth. ${ }^{9}$ Benefiting from printing technology, various electronic components on paper substrates have been developed, such as transistors, ${ }^{10}$ memory, ${ }^{11}$ detectors, ${ }^{12}$ capacitors, ${ }^{13}$ resistors $^{14}{ }^{14}$ diodes, ${ }^{15}$ and power generators, ${ }^{16}$ which by integrating these elements into functional circuits enable advanced applications, including nanopaper antennas, ${ }^{17}$ paper-based lithium-ion batteries, ${ }^{18}$ and radio frequency identification tags. ${ }^{19}$

However, due to the extremely low thermal conductivity of plastic and paper substrates, ${ }^{20,21}$ heat dissipation problems exist in both device types, making it difficult for them to function at high temperatures. ${ }^{22}$ Additionally, when the dimensions of the device are smaller, as is the trend of modern integrated electronics, ${ }^{23}$ the decrease in the contact areas between the electronic components and the substrate results in an increase in device power density. This causes heat accumulation to occur during operation, degrading the flexible device performance. ${ }^{24-26}$ Moreover, the thermal issue is especially critical for flexible optoelectronics. ${ }^{27}$ Regardless of whether the application involves light harvesting (such as photovoltaic cells and photosensors) or irradiation (such as light-emitting diodes and lasers), a considerable amount of thermal energy accompanying the light can decrease the device performance significantly. ${ }^{28}$ Therefore, improving heat dissipation is the next requirement for high precision and performance flexible electronic applications.

Recently, due to immunity from solar interference, solar-blind deep-ultraviolet (DUV) photodetectors (PDs) have been widely used in various applications, including automatization, intersatellite and underwater communications, biological sterilization detectors, and military detection. ${ }^{29,30}$ These applications usually involve tasks requiring high sensitivity and security. Therefore, stable and high-performance devices are required, thus also

\footnotetext{
${ }^{1}$ Computer, Electrical, and Mathematical Sciences and Engineering (CEMSE) Division, King Abdullah University of Science \& Technology (KAUST), Thuwal 23955-6900, Saudi Arabia and ${ }^{2}$ University of Maryland Energy Research Center, and Department of Materials Science and Engineering, University of Maryland, College Park, MD 20742, USA Correspondence: J-H. He (jrhau.he@kaust.edu.sa)
}

Received: 17 March 2018 Revised: 23 June 2018 Accepted: 4 July 2018

Published online: 16 August 2018 
necessitating strong thermal management in order to dissipate the heat generated by the high energy DUV light.

To achieve these goals, we fabricated high thermally conductive boron nitride (BN) paper using 2D hexagonal (h)-BN nanosheets structurally supported by 1D nanofibrillated cellulose (NFC). This flexible BN paper exhibits superior thermal conductivity that is three orders of magnitude higher than conventional plastic or paper substrates, making it an ideal dielectric substrate to realize flexible electronics with high thermal stability. Owing to the highquality fabrication of combining nano-sized h-BN and NFC materials, the BN nanopaper possesses a smooth surface, enabling the use of conventional microelectronic deposition processes for device fabrication, such as sputter deposition and E-beam evaporation. Flexible DUV PD devices were constructed using sputtered Pt electrodes on the BN paper substrate and demonstrated remarkable photosensing performance (detectivity of up to $8.05 \times 10^{10} \mathrm{~cm} \mathrm{~Hz}^{1 / 2} / \mathrm{W}$ ) and fast photoresponse (response and recovery times were $0.267 \mathrm{~s}$ and $0.393 \mathrm{~s}$, respectively). The flexible

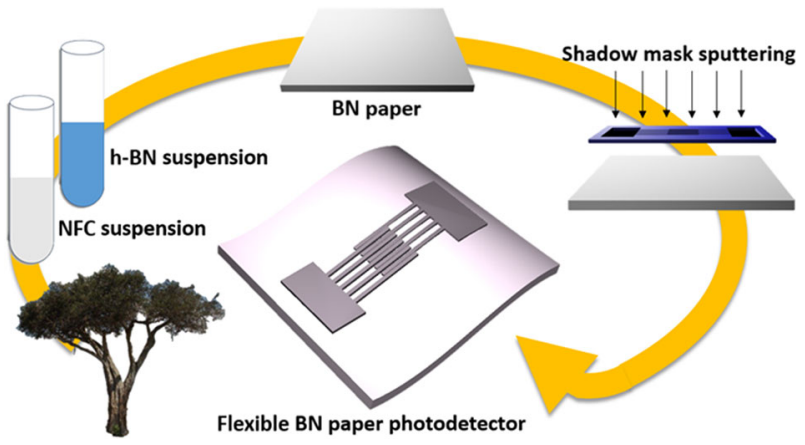

Fig. 1 Schematic of the fabrication process of the flexible BN paper PD
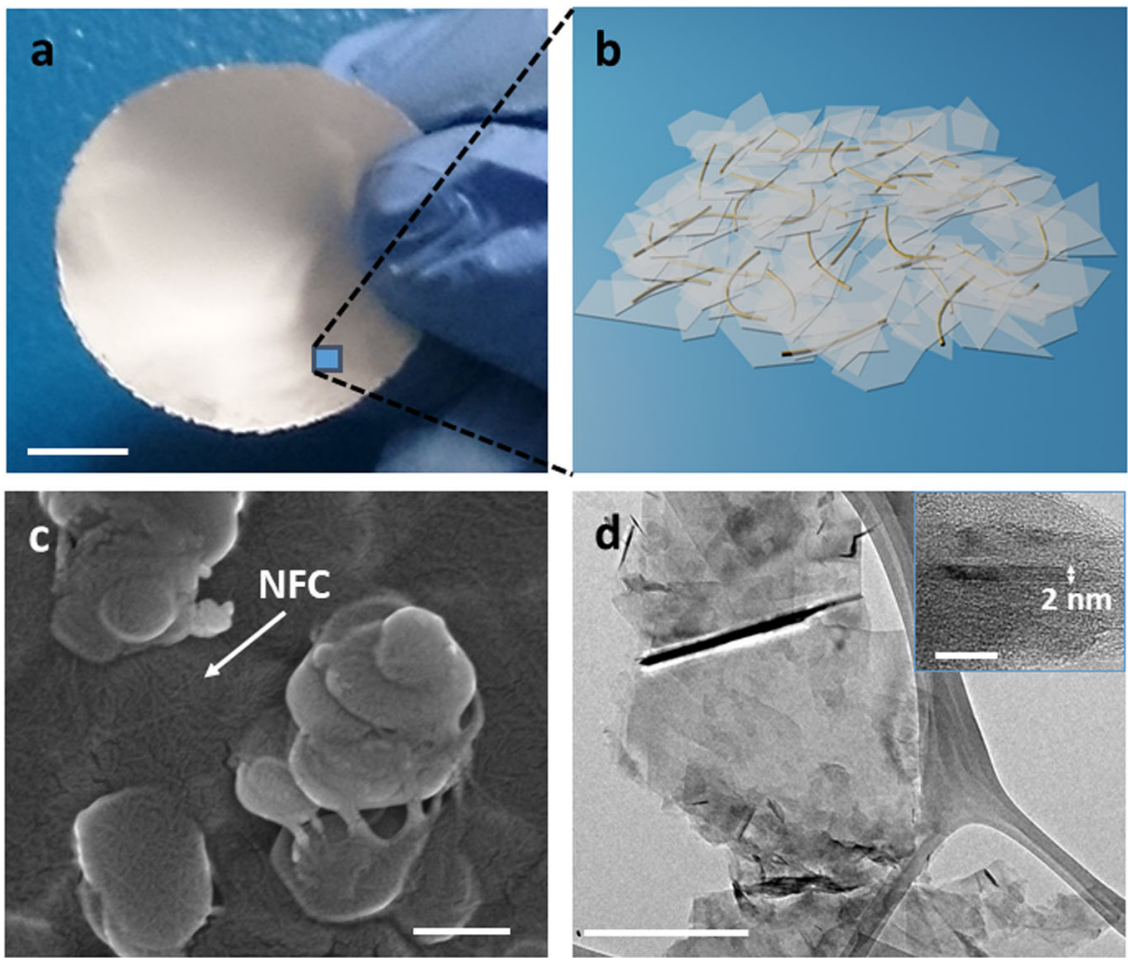

Fig. 2 Surface morphology and structure of the BN paper. a Optical image of the BN paper. The scale bar is $3 \mathrm{~cm}$. $\mathbf{b}$ Structure illustration of the BN paper, which is composed of 2D h-BN and 1D NFC. c SEM image of the BN paper surface, showing how the h-BN nanosheets are structural supported by the NFC fibers (scale bar $=300 \mathrm{~nm}$ ). d TEM image showing a top view of the h-BN nanosheets (scale bar $=500 \mathrm{~nm}$ ). The inset is the cross-section of a h-BN nanosheet, demonstrating its narrow thickness (scale bar $=10 \mathrm{~nm}$ ) 
a

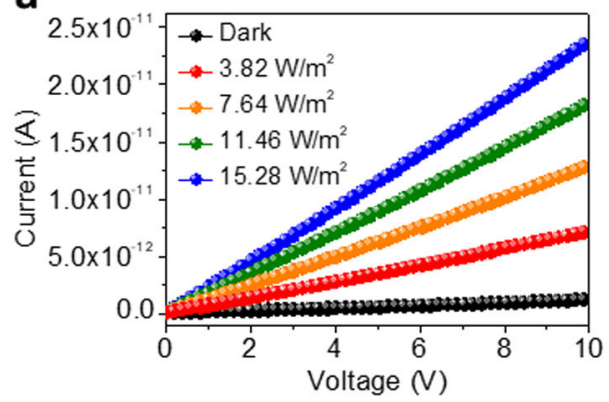

C

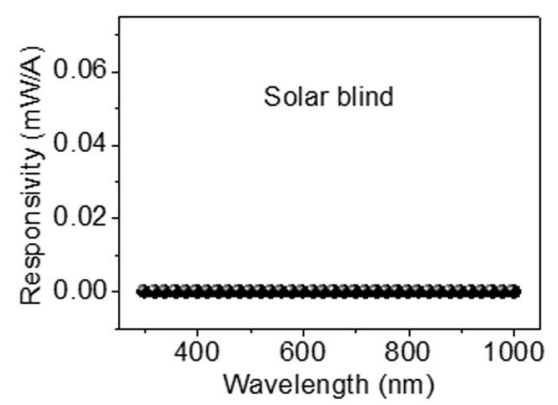

d

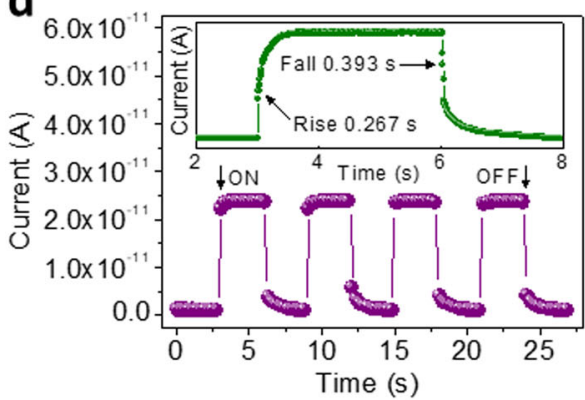

b

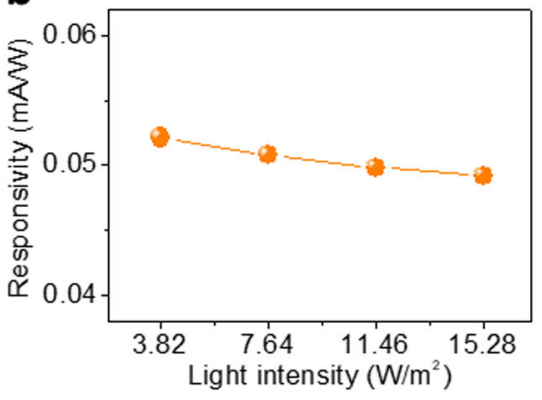

e
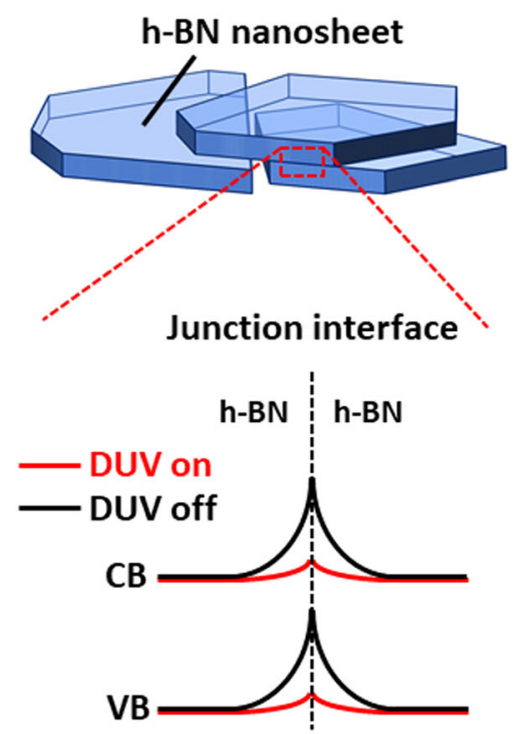

Fig. 3 The photodetection characteristics of the BN paper PD. a I-V curves of a BN paper PD under dark and $185 \mathrm{~nm}$ DUV illumination at different light intensities. b Responsivity of the BN paper PD as a function of DUV light intensity. c Spectral-dependent responsivity of the BN paper PD to show its solar-blind behavior. $\mathbf{d}$ The device's current as a function of time for ON/OFF switching between dark and $185 \mathrm{~nm}$ light illumination. The inset shows the high-resolution time response under the same conditions to verify the rise and fall times of the BN paper PD. e Illustration of the junction barrier between h-BN nanosheets under DUV ON and DUV OFF conditions. CB and VB are the conduction and valence bands of $\mathrm{h}-\mathrm{BN}$

as surveyed by atomic force microscopy (AFM; Fig. S2 in the Supplementary Information). As a result, devices based on BN paper are compatible with semiconductor fabrication processes, such as E-beam evaporation or sputtering, thus enabling mass production. Furthermore, the smooth surface of BN paper also enables scalable patterning fabrication methods. As shown in Fig. S3, the patterned Au electrodes were successfully constructed on BN paper using electron beam lithography process, which is impossible for conventional paper. However, we found the flexible nature of $\mathrm{BN}$ paper can lead to the pattern distortion during lithography. Although this pattern distortion is not obvious for fabricating microscale patterns, it might limit the BN paper for nanoelectronics applications using solution-based lithography.

An optical image and illustrated structure of the BN paper is shown in Fig. 2a, b. In this structure, the 2D h-BN nanosheets stack randomly along the in-plane direction of the paper. The large contact area between the h-BN nanosheets helps assist in heat dissipation. Owing to the minimized thermal contact resistance between the h-BN sheets and the outstanding thermal conductivity of h-BN, the BN paper is a suitable substrate for improving thermal management in flexible electronics. The surface morphology of the BN paper is shown in the scanning electron microscopy
(SEM) image in Fig. 2c, which demonstrates that the 2D h-BN material is compactly wired by the 1D NFC, which provides strong mechanical strength and durability for the paper structure. On the other hand, the ultrathin nature of the 2D h-BN nanosheets, as demonstrated by the transmission electron microscopy (TEM) in Fig. $2 \mathrm{~d}$, helps maintain the flexibility of the BN paper, as well as providing its optoelectronic functionality. The electron diffraction pattern of an h-BN nanosheet is shown in Fig. S4, revealing its high crystallinity.

For solar-blind detection, the PD cannot absorb above $280 \mathrm{~nm}$, rather only responding to wavelengths shorter than solar radiation. In our PD design, NFC is not conductive and purely provides mechanical strength and flexibility for the BN paper. On the other hand, besides greatly improving the thermal conductivity of the paper, the h-BN also acts as the active material. With a dielectric constant of $3-4, \mathrm{~h}-\mathrm{BN}$ is not very conductive. ${ }^{34}$ However, due to the large bandgap of $5.97 \mathrm{eV}^{35} \mathrm{~h}-\mathrm{BN}$ is able to absorb light in the DUV wavelengths to produce a large photogenerated current. Based on this mechanism, h-BN composed PDs exhibit an outstanding capability for solar-blind detection.

Figure 3a demonstrates the current-voltage $(I-V)$ characteristics of a BN paper PD in the dark and under $185 \mathrm{~nm}$ DUV illumination 
at different light intensities. In this work, the typical measurement conditions were under a bias of $10 \mathrm{~V}$ unless otherwise noted. The current varied with light intensity, which demonstrates the high sensitivity of the devices for detecting DUV light. We estimated the responsivity $(R)$ of the BN paper PD using the equation $R=I_{\mathrm{p}} /$ $P$, where $I_{\mathrm{p}}$ is the photogenerated current, and $P$ is the UV illumination power. ${ }^{36}$ The light intensity-dependent responsivity at $10 \mathrm{~V}$ bias is shown in Fig. 3b, which indicates that the responsivity slightly decreases with light intensity. The reason for such light intensity-dependent responsivity is that the excess number of photogenerated carriers produced when the device is under high-intensity illumination will raise the possibility of carrier recombination. ${ }^{37}$ To further examine the solar-blind property of the BN paper PD, we performed wavelength-dependent measurements on the device from 300 to $1000 \mathrm{~nm}$. The results shown in Fig. $3 c$ demonstrate that the BN paper PD has no response to this region of the solar black-body spectrum, confirming its solar-blind characteristics. Time-resolved study is also important for determining the practicality of the PD. Figure $3 d$ presents the current of the BN paper PD as the $185 \mathrm{~nm}$ DUV light is switched ON and OFF over time. The reversible current switching demonstrates the reliable photodetection ability of the BN paper device. Furthermore, it also indicates short response and recovery times of $0.267 \mathrm{~s}$ and $0.393 \mathrm{~s}$, respectively, as shown in the Fig. $3 \mathrm{~d}$ inset to illustrate the device's fast photodetection. Previously, an h-BN film PD has been reported that featured a recovery time of a few seconds. ${ }^{38}$ Therefore, the much shorter recovery time of the $\mathrm{BN}$ paper PD in this study implies that an additional carrier transport mechanism is involved.

Because the current flow in the $B N$ paper PD involves connections between many h-BN nanosheets, the junction interface between these nanosheets will play a key role in the carrier transport. The schematic of Fig. 3e illustrates the additional conducting mechanism observed in the BN paper device. When $\mathrm{h}-$ BN nanosheets contact with each other, a depletion layer emerges at the junction interface due to the surface defects of the material. ${ }^{39-41}$ Under the dark condition, the barrier at the h-BN nanosheet-nanosheet interface due to the depletion layer will hinder carrier transport in the device, dominating the transport of the overlapping nanosheets. This leads to a very low dark current in the BN paper PD. On the other hand, under DUV illumination, large amounts of electron-hole pairs are generated in h-BN. The significantly increased carrier density can decrease the thickness of the depletion layer, thus reducing the junction barrier height and benefiting carrier transport. ${ }^{42}$ When the illumination source is turned OFF, the fast electron-hole recombination raises the junction barrier to the original level immediately, leading to the short recovery time of the current in the BN paper PD. With the fast photoresponse, the device is able to achieve efficient detection of various intensities of light.

Detectivity $\left(D^{*}\right)$ is the key factor for judging the sensitivity of PDs in terms of their ability to detect an optical signal. ${ }^{43}$ Because of a lack of background noise from nature in the solar-blind wavelength region, detectors with a detectivity higher than $10^{8}$ $\mathrm{cm} \mathrm{Hz}{ }^{1 / 2} / \mathrm{W}$ can usually achieve excellent solar-blind photodetection. ${ }^{44}$ In this study, the BN paper PD possesses a detectivity of $8.05 \times 10^{10} \mathrm{~cm} \mathrm{~Hz}^{1 / 2} / \mathrm{W}$ at $10 \mathrm{~V}$ bias, based on the equation $D^{*}=1 /$ $\mathrm{NEP}=f^{1 / 2} / P=R /\left(2 e J_{\mathrm{d}}\right)^{1 / 2}$, in which NEP is the noise-equivalent power, $f$ is the frequency bandwidth of the PD, $e$ is the charge of the electron, and $J_{\mathrm{d}}$ is the dark current density. ${ }^{45}$ Such a high detectivity value confirms the practicality of the BN paper PD for reliable solar-blind detection.

Strong thermal management can significantly improve device performance, but a problem with flexible electronics today is the poor heat dissipation of convention flexible substrates. For example, plastic substrates have low thermal conductivity of around $0.27 \mathrm{~W} / \mathrm{mK}$, and typical paper substrates are $0.04 \mathrm{~W} /$ $\mathrm{mK}^{20,21}$ In contrast, the thermal conductivity of BN paper has
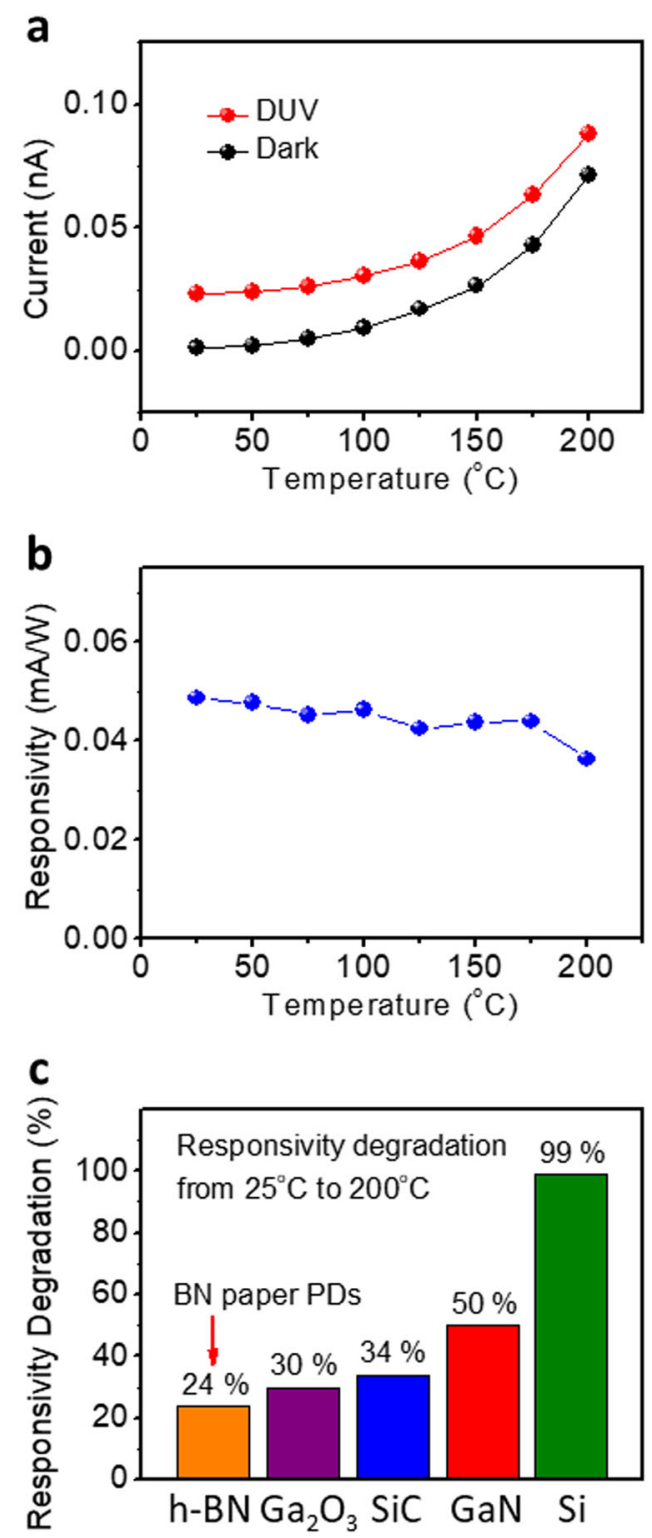

Fig. 4 The photodetection performance of the $B N$ paper PD at temperatures ranging from 25 to $200^{\circ} \mathrm{C}$. a Temperature dependence of the current under dark and $185 \mathrm{~nm}$ DUV light at an intensity of $15.28 \mathrm{~mW} / \mathrm{cm}^{2}$ and $10 \mathrm{~V}$ bias. b The responsivity as a function of temperature under $185 \mathrm{~nm}$ DUV illumination at an intensity of $15.28 \mathrm{~mW} / \mathrm{cm}^{2}$ and $10 \mathrm{~V}$ bias. c Comparison of the responsivity degradation from 25 to $200^{\circ} \mathrm{C}$ for different PDs made of BN paper, $\beta$ $\mathrm{Ga}_{2} \mathrm{O}_{3}{ }^{48} \mathrm{n}-\mathrm{p}-\mathrm{n} \mathrm{SiC}{ }^{47} \mathrm{GaN}^{49}$ and $\mathrm{n}-\mathrm{p}-\mathrm{n} \mathrm{Si}{ }^{47}$

been measured as high as $146 \mathrm{~W} / \mathrm{mK}$ using steady-state method, ${ }^{46}$ which is three orders larger than conventional flexible substrates. Based on the high thermally conductive structure of the h-BN network, the heat generated in the BN paper PD can be easily dissipated and thus enabling the device to be more thermally stable.

To highlight the thermal stability of the BN paper PD, we investigated the device performance from 25 to $200^{\circ} \mathrm{C}$. Figure $4 a$ demonstrates the temperature-dependent $I-V$ curves of the device under dark and $185 \mathrm{~nm}$ light illumination. The increasing current with temperature implies the insulating behavior of h-BN, which means more carriers are thermally excited to the conduction band at high temperature, leading to the enhanced 
a

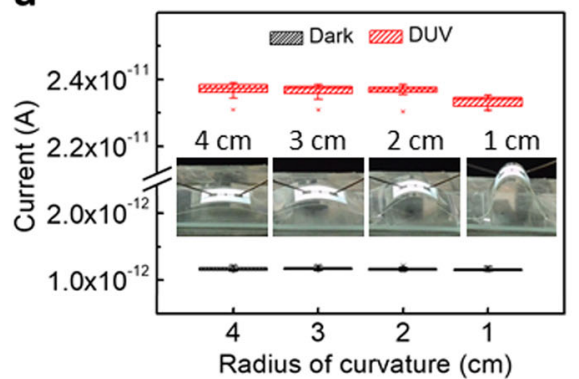

b

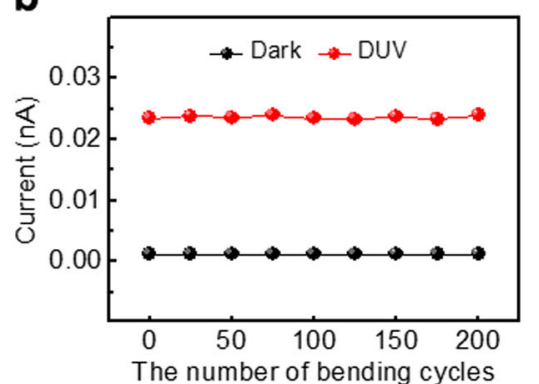

Fig. 5 The flexibility and durability of the BN paper PD. a The DUV light ON/OFF distribution of a BN paper PD as a function of bending radius of curvature. The inset shows images of the device under different bending conditions. $\mathbf{b}$ The ON/OFF states of the BN paper PD as a function of bending cycles. A cycle starts from an unbent state to a bending radius of $1 \mathrm{~cm}$, and back again to the unbent state

current. The responsivity of the BN paper PD from 25 to $200^{\circ} \mathrm{C}$ at $10 \mathrm{~V}$ bias is shown in Fig. 4b. Because the responsivity of the device increases with biased voltage, we fixed the bias on $10 \mathrm{~V}$ to evaluate the device performance at different temperatures. Even at a temperature of $200^{\circ} \mathrm{C}$, the responsivity of the $\mathrm{BN}$ paper PD is only $24 \%$ less compared to at room temperature, which demonstrates the steady photodetection ability of the device at different temperatures. The high-temperature operation of the BN paper PD compared to several PDs based on other semiconductors $\left(\beta-\mathrm{Ga}_{2} \mathrm{O}_{3}, \mathrm{SiC}, \mathrm{GaN}\right.$, and $\left.\mathrm{Si}\right)$ is demonstrated in Fig. 4c. The $n-p-n$ Si PD almost entirely (>99\%) loses its detection capability at $200{ }^{\circ} \mathrm{C}^{47}$ Compared with other rigid material-based DUV detectors, including $\mathrm{Ga}_{2} \mathrm{O}_{3}{ }^{48} \mathrm{SiC}^{47}$ and $\mathrm{GaN}_{1}{ }^{49}$ the $\mathrm{BN}$ paper $\mathrm{PD}$ displays the smallest responsivity degradation from 25 to $200^{\circ} \mathrm{C}$, thus confirming its outstanding thermal stability.

Durability is also important for practical flexible and wearable applications. We performed a bending test to examine the performance of the BN paper PD under different radius of curvatures, as shown in Fig. 5a. In this test, the device was operated under dark and $185 \mathrm{~nm}$ DUV illumination at a bias of $10 \mathrm{~V}$ and the bending condition was described by the radius of curvature between the two edges of the device. The consistent device performance at the same dark current and photocurrent levels in different bending conditions provides convincing evidence for the high flexibility and stability of the BN paper PD. Moreover, the endurance test shown in Fig. 5b was executed to verify the mechanical robustness of the device. After being bent 200 times at a radius of $1 \mathrm{~cm}$, the device performance did not show significant change, confirming the high durability of the NFC supported BN paper PD.

In summary, the strategy described herein suggests a method for improving thermal management in flexible electronics. By incorporating 2D h-BN nanosheets and 1D cellulose nanofibers, we were able to achieve a flexible BN paper substrate that features a high thermal conductivity $(146 \mathrm{~W} / \mathrm{mK})$ and smooth surface (Rms $=122 \mathrm{~nm}$ ), which facilitates microelectronic fabrication processes. The flexible DUV paper PD was demonstrated with superior properties, including an outstanding detectivity (up to $8.05 \times 10^{10}$ $\mathrm{cm} \mathrm{Hz}^{1 / 2} / \mathrm{W}$ ), fast photoresponse (response and recovery times of $0.267 \mathrm{~s}$ and $0.393 \mathrm{~s}$, respectively), high-temperature tolerance $\left(200^{\circ} \mathrm{C}\right)$, and mechanical robustness (demonstrating reproducible photodetection over 200 bending cycles), making this device promising in solar-blind flexible applications. Additionally, because of the dielectric feature in the normal visible light wavelength region, BN paper is also an ideal flexible substrate for other DUVexcluded flexible applications. Using standard semiconductor deposition methods, high-quality semiconductor materials and metal electrodes can be constructed on BN paper, which enables high-performance flexible electronics with excellent thermal management.

\section{METHODS}

Device fabrication

To make the BN paper substrates, a $5 \mathrm{mg} / \mathrm{mL} \mathrm{h}$-BN solution was made by adding h-BN micropowder (Graphene Supermarket Inc.) into isopropyl alcohol by $48 \mathrm{~h}$ sonication. The h-BN dispersion was then purified using a centrifuge at a spin rate of $1000 \mathrm{rpm}$ for $15 \mathrm{~min}$. The oxidation process was carried out by adding $78 \mathrm{mg}$ of 2,2,6,6-tetramethylpiperidine-1-oxyl and $514 \mathrm{mg} \mathrm{NaBr}$ to $5 \mathrm{~g}$ kraft bleached softwood pulp into $12 \% \mathrm{NaClO}$ solution with a $\mathrm{pH}$ of 10.5 (controlled by adding $\mathrm{NaOH}$ ). ${ }^{31}$ After oxidation, deionized water was added to the oxidized product to prepare a $1 \mathrm{wt} \%$ cellulose suspension. The suspension was then processed by a microfluidizer ( $M$ $110 \mathrm{EH}$, Microfluidics Ind.) to achieve the fine NFC suspension (diluted to $0.2 \mathrm{wt} \%$ with deionized water). The h-BN and NFC suspensions were mixed together by sonication for $15 \mathrm{~min}$ at a ratio of 1:1 and then filtered through a membrane filter (MF-Millipore) featuring $0.65 \mu \mathrm{m}$ pore size. The BN paper was obtained by drying the wet film on the filter using a sequence of mechanical pressing methods, as previously described. ${ }^{50}$ Finally, 200-nm thick Pt electrodes were deposited on the BN paper surface using shadow mask sputtering to form the BN paper PDs.

\section{Experiments}

The SEM images of the BN paper surface were taken using a Nova Nano 630 SEM with an accelerating voltage of $5 \mathrm{kV}$. The quality of the h-BN nanosheets was investigated using a JEOL JEM-2100 TEM at a voltage of $200 \mathrm{kV}$. The surface roughness of the BN paper was examined with a commercial AFM (Cypher ES AFM, Asylum Research Oxford Instruments) using Ti/Pt-coated silicon cantilevers (Asylum research). The tip curvature radius was $\sim 28 \mathrm{~nm}$, and the resonance frequency was $\sim 75 \mathrm{kHz}$. The $I-V$ characteristics of the BN paper PDs were surveyed using a Keithley 4200SCS semiconductor characterization system in combination with an EverBeing Cryogenic Probe Station CG-196-200 for controlling the temperature. The device was annealed on a hotplate at $100^{\circ} \mathrm{C}$ for $2 \mathrm{~min}$ before $I-V$ measurements. Unless otherwise noted, the measurements were performed using an applied bias of $10 \mathrm{~V}$. For the bending test in Fig. $5 a$, we kept the device on different bending conditions and collected the data. For durability test in Fig. $5 \mathrm{~b}$, we collected a data point (at unbent state) after every 25 bending cycles. When bending radius smaller than $0.5 \mathrm{~cm}$, the mechanical stress can result in permanent damage on $\mathrm{BN}$ paper device. For electron beam lithography patterning, the BN paper was fixed on a rigid $\mathrm{Si}$ substrate by tape, and used an electron beam system (Crestec, CABL-9000C series) to perform the experiment.

Data availability

The data sets generated during and/or analyzed during the current study are available from the corresponding author on reasonable request.

\section{ACKNOWLEDGEMENTS}

This work was financially supported by the King Abdullah University of Science and Technology (KAUST) Office of Sponsored Research (OSR) (OSR-2016-CRG5-3005), KAUST solar center (FCC/1/3079-08-01), and KAUST baseline funding. 


\section{AUTHOR CONTRIBUTIONS}

C.-H.L. and J.-H.H. conceived the experiments. C.-H.L., H.-C.F., and M.-L.T. executed the measurements. C.-H.L. and H.-C.F. designed the figures. C.-H.L., W.L., L.Z., S.-H.J., and L. H. fabricated the BN paper and photodetectors. C.-H.L. and J.-H.H. performed the data analysis. C.-H.L., B.C., and J.-H.H. wrote the manuscript. All authors discussed the results and commented on the manuscript.

\section{ADDITIONAL INFORMATION}

Supplementary information accompanies the paper on the npj 2D Materials and Applications website (https://doi.org/10.1038/s41699-018-0070-6).

Competing interests: The authors declare no competing interests.

Publisher's note: Springer Nature remains neutral with regard to jurisdictional claims in published maps and institutional affiliations.

\section{REFERENCES}

1. Kim, D. H., Xiao, J. L., Song, J. H., Huang, Y. G. \& Rogers, J. A. Stretchable, curvilinear electronics based on inorganic materials. Adv. Mater. 22, 2108-2124 (2010).

2. Rogers, J. A., Someya, T. \& Huang, Y. G. Materials and mechanics for stretchable electronics. Science 327, 1603-1607 (2010).

3. Kim, D. H. et al. Stretchable and foldable silicon integrated circuits. Science $\mathbf{3 2 0}$, 507-511 (2008).

4. Zhan, Y., Mei, Y. \& Zheng, L. Materials capability and device performance in flexible electronics for the internet of things. J. Mater. Chem. C 2, 1220-1232 (2014).

5. Axisa, F. et al. Flexible technologies and smart clothing for citizen medicine, home healthcare, and disease prevention. IEEE Trans. Inf. Technol. Biomed. 9, 325-336 (2005).

6. NK Wood Research. Global Flexible Electronics Market Forecast 2017-2024. https:// www.inkwoodresearch.com/reports/global-flexible-electronics-market-forecast2017-2024 (2016).

7. Wong, W. S. \& Salleo, A. Flexible Electronics: Materials and Applications (Springer Science \& Business Media, New York, 2009).

8. Russo, A. et al. Pen-on-paper flexible electronics. Adv. Mater. 23, 3426-3430 (2011).

9. Tobjörk, D. \& Österbacka, R. Paper electronics. Adv. Mater. 23, 1935-1961 (2011).

10. Kim, Y. H., Moon, D. G. \& Han, J. I. Organic TFT array on a paper substrate. IEEE Electron Device Lett. 25, 702-704 (2004).

11. Lien, D. H. et al. All-printed paper memory. ACS Nano 8, 7613-7619 (2014).

12. Lin, C. H. et al. Highly deformable origami paper photodetector arrays. ACS Nano 11, 10230-10235 (2017).

13. Yang, L. et al. Hierarchical network architectures of carbon fiber paper supported cobalt oxide nanonet for high-capacity pseudocapacitors. Nano Lett. 12, 321-325 (2012).

14. Siegel, A. C. et al. Foldable printed circuit boards on paper substrates. Adv. Funct. Mater. 20, 28-35 (2010).

15. Martins, Ferreira, I. \& Fortunato, E. Electronics with and on paper. Phys. Status Solidi. 5, 332-335 (2011)

16. Yuan, L. et al. Paper-based supercapacitors for self-powered nanosystems. Angew. Chem. 124, 5018-5022 (2012).

17. Nogi, M., Komoda, N., Otsuka, K. \& Suganuma, K. Foldable nanopaper antennas for origami electronics. Nanoscale 5, 4395-4399 (2013).

18. Cheng, Q. et al. Folding paper-based lithium-ion batteries for higher areal energy densities. Nano Lett. 13, 4969-4974 (2013).

19. Yang, L., Rida, A., Vyas, R. \& Tentzeris, M. M. RFID tag and RF structures on a paper substrate using inkjet-printing technology. IEEE Trans. Microw. Theory Tech. 55, 2894-2901 (2007)

20. Kymäläinen, H.-R. \& Sjöberg, A.-M. Flax and hemp fibres as raw materials for thermal insulations. Build. Environ. 43, 1261-1269 (2008).

21. Weidenfeller, B., Höfer, M. \& RSchilling, F. Thermal conductivity, thermal diffusivity, and specific heat capacity of particle filled polypropylene. Compos. A 35, 423-429 (2004).

22. Forrest, S. R. \& Thompson, M. E. Introduction: organic electronics and optoelectronics. Chem. Rev. 107, 923-925 (2007).

23. Ferain, I., Colinge, C. A. \& Colinge, J.-P. Multigate transistors as the future of classical metal-oxide-semiconductor field-effect transistors. Nature 479, 310-316 (2011).

24. Pop, E., Sinha, S. \& Goodson, K. E. Heat generation and transport in nanometerscale transistors. Proc. IEEE 94, 1587-1601 (2006).
25. Moore, A. L. \& Shi, L. Emerging challenges and materials for thermal management of electronics. Mater. Today 17, 163-174 (2014).

26. Garimella, S. V. et al. Thermal challenges in next-generation electronic systems. IEEE Trans. Compon. Packag. Technol. 31, 801-815 (2008).

27. Kim, T. I. et al. Injectable, cellular-scale optoelectronics with applications for wireless optogenetics. Science 340, 211-216 (2013).

28. Kim, L., Choi, J. H., Jang, S. H. \& Shin, M. W. Thermal analysis of LED array system with heat pipe. Thermochim. Acta 455, 21-25 (2007).

29. Tsai, D. S. et al. Solar-blind photodetectors for harsh electronics. Sci. Rep. 4, 2628 (2013).

30. Ozbay, E. et al. High-performance solar-blind photodetectors based on AlxGa1-xN heterostructures. IEEE J. Sel. Top. Quant. Electron. 10, 742-751 (2004).

31. Zhu, H. et al. Biodegradable transparent substrates for flexible organic-lightemitting diodes. Energy Environ. Sci. 6, 2105-2111 (2013).

32. $\mathrm{Zhu}, \mathrm{H}$. et al. Highly thermally conductive papers with percolative layered boron nitride nanosheets. ACS Nano 8, 3606-3613 (2014).

33. Xu, R., Fleming, P. D., Pekarovicova, A. \& Bliznyuk, V. The effect of ink jet paper roughness on print gloss. J. Imaging Sci. Technol. 49, 660-666 (2005).

34. Young, A. F. et al. Electronic compressibility of layer-polarized bilayer graphene. Phys. Rev. B 85, 235458 (2012).

35. Dean, C. R. et al. Boron nitride substrates for high-quality graphene electronics. Nat. Nanotechnol. 5, 722-726 (2010).

36. Wei, T. C. et al. See-through Ga2O3 solar-blind photodetectors for use in harsh environments. IEEE J. Sel. Top. Quant. Electron. 20, 3802006 (2014).

37. Saleh, B. E. \& Teich, M. C. Fundamentals of Photonics (Wiley, New York, 1991).

38. Sajjad, M., Jadwisienczak, W. M. \& Feng, P. Nanoscale structure study of boron nitride nanosheets and development of a deep-UV photodetector. Nanoscale $\mathbf{6}$, 4577-4582 (2014).

39. Auwärter, W., Muntwiler, M., Osterwalder, J. \& Greber, T. Defect lines and twodomain structure of hexagonal boron nitride films on Ni (111). Surf. Sci. 545, L735-L740 (2003).

40. Lyalin, A., Nakayama, A., Uosaki, K. \& Taketsugu, T. Adsorption and catalytic activation of the molecular oxygen on the metal supported h-BN. Top. Catal. 57, 1032-1041 (2014).

41. Preobrajenski, A. B. et al. Adsorption-induced gap states of h-BN on metal surfaces. Phys. Rev. B 77, 085421 (2008).

42. Sze, S. M. \& Ng, K. K. Physics of Semiconductor Devices (John Wiley \& Sons, Hoboken, NJ, 2006).

43. Jones, R. C. Phenomenological description of the response and detecting ability of radiation detectors. Proc. IRE 47, 1495-1502 (1959).

44. Zeller, J. W. et al. Development of Ge PIN photodetectors on $300 \mathrm{~mm}$ Si wafers for near-infrared sensing. Int. J. Eng. Res. Technol. 8, 23-33 (2015).

45. Tsai, D. S. et al. Few layer MoS2 with broadband high photogain and fast optical switching for use in harsh environments. ACS Nano 7, 3905-3911 (2013).

46. Yang, B., Liu, W. L., Liu, J. L., Wang, K. L. \& Chen, G. Measurements of anisotropic thermoelectric properties in superlattices. Appl. Phys. Lett. 81, 3588-3590 (2002).

47. Wu, K. H. et al. A high optical-gain/spl beta/-SiC bulk-barrier phototransistor for high-temperature applications. IEEE Photonics Technol. Lett. 10, 1611-1613 (1998).

48. Zou, R. et al. High-detectivity nanowire photodetectors governed by bulk photocurrent dynamics with thermally stable carbide contacts. Nanotechnology 24, 495701 (2013)

49. Vittorio, M. D. et al. High temperature characterization of GaN-based photodetectors. Sens. Actuator A Phys. 113, 329-333 (2004).

50. Zhu, H., Fang, Z., Preston, C., Li, Y. \& Hu, L. Transparent paper: fabrications, properties, and device applications. Energy Environ. Sci. 7, 269-287 (2014).

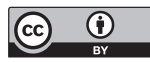

Open Access This article is licensed under a Creative Commons Attribution 4.0 International License, which permits use, sharing, adaptation, distribution and reproduction in any medium or format, as long as you give appropriate credit to the original author(s) and the source, provide a link to the Creative Commons license, and indicate if changes were made. The images or other third party material in this article are included in the article's Creative Commons license, unless indicated otherwise in a credit line to the material. If material is not included in the article's Creative Commons license and your intended use is not permitted by statutory regulation or exceeds the permitted use, you will need to obtain permission directly from the copyright holder. To view a copy of this license, visit http://creativecommons. org/licenses/by/4.0/.

(c) The Author(s) 2018 